
Wang*

\title{
Feature extraction for license plate location based on $L_{0}$-norm smoothing
}

https://doi.org/10.1515/comp-2019-0007

Received July 10, 2018; accepted May 16, 2019

\begin{abstract}
We propose a simple feature extraction algorithm for license plate location, which can reduce the occurrence of pseudo-licenses significantly. Our scheme arises from a novel $L_{0}$-norm image smoothing, in which the multiple local textures in the complex backgrounds can be suppressed remarkably without changing the structures and edges of the license objects. Due to this "edgeaware" property, we then combine a feature filtering with an efficient binarized image, a simple multi-scale image analysis algorithm, to remove the potential false license plates. Finally, we extract license plates with a projection method. Experimental results show the proposed method provides a flexible and powerful way to the license plate location in complex backgrounds.
\end{abstract}

Keywords: license plate location, $L_{0}$-norm minimization, feature filtering, binarized image

\section{Introduction}

License plate location plays an important role in a license plate recognition system. As a basis of license character recognition, the accuracy and robustness of license location directly determine the performance of the entire li-

\footnotetext{
Junqing Huang: Mathematics and System Science, Beihang University (BUAA), China; E-mail: zcfeng@buaa.edu.cn

Michael Ruzhanshy: University of Ghent, Belgium; Queen Mary University of London, UK; Department of Mathematics, Imperial College London, UK; E-mail: ruzh@ic.ac.uk Haoxiang Feng: Mathematics and System Science, Beihang University (BUAA), China; E-mail: HaoXiang_Feng@buaa.edu.cn Lingfang Zheng: Mathematics and System Science, Beihang University (BUAA), China; E-mail: LINGFANGbeibei@buaa.edu.cn Xin Huang: Mathematics and System Science, Beihang University (BUAA), China; E-mail: 17683746951@163.com

*Corresponding Author: Haihui Wang: Mathematics and System Science, Beihang University (BUAA), China;

E-mail: whhmath@buaa.edu.cn
}

cense plate recognition system. In many practical applications, license plate recognition systems typically work outdoors, and the captured images inevitably suffer from different kinds of degradations such as light, weather and complex backgrounds. Such diversity, uncertainty and complexity of environment give rise to the challenge of the license plate location; and the problem in such cases has attracted wide attention both academically and industrially.

During the past few decades, different kinds of license plate location methods have been proposed, which can be broadly categorized into two themes: feature-based and learning-based methods [1]. However, it is still a challenging problem to give a robust solution in complex backgrounds, especially when the environment changes drastically, the accuracy of most methods would drop significantly in such cases. For example, the projection-based algorithms [2, 3] are simple, but they commonly rely on complex threshold settings and may encounter false detections. The texture-based methods $[4,5]$ try to discriminate licenses from scene background based on the textual information, including car lights, grid and heat sinks, but they may suffer from the same deficits as the projection-based ones for their high sensitivity of the fake license targets. At the same time, the color-based methods [6-8] are also vulnerable to the change of lighting, weather and color in both car body and the complex background. In consideration of the practical situations, some methods [1,9-11] have combined all above features to strengthen the whole location system; nevertheless, these methods are computationally complex, especially when many edges occurs in the complex circumstance or varying illumination conditions.

On the other hand, learn-based methods have been well studied in object detection and recognition tasks. These methods [12-16] tend to extract license plates using neural networks (NNs) as filters for analyzing the color and texture properties of license plates. More recently, strongly benefiting from the convolutional neural networks (CNN) [17, 18], different architectures are springing out, which in fact, can identify license plate precisely 
and are considered to be the leading techniques for license plate detection. Yet, one bottleneck of these learning methods is the need for supervision that requires mass high-quality matched training pairs of samples in practice.

Considering the complex scene, researchers have been long eager to design a license plate location algorithm with high precision and robustness. In this paper, we propose a simple location method based on an "edgeaware" image decomposition. We first introduce a novel $L_{0}$-norm image smoothing to separate local image textures from structures. Since such a decomposition has an edgeaware property, the multiple local textures can be removed from the complex background and licenses with salient edges are highlighted and easy to extract. In view of this, we then introduce a filtering process on binarized images to further delete the potential false license plates. Because the binarized image provides an efficient way to solve the scale problem of the licenses; we finally extract the license plate with a simple projection location method. We demonstrate the versatility of this scheme and show that our method provides a rather flexible and powerful way to license plate location, especially in complex backgrounds.

\section{$2 L_{0}$-norm minimization}

In recent years, "edge-aware" image decomposition has received extensive attention, providing a sparse solution for many image processing problems. In [19], Xu proposed a $L_{0}$-norm smoothing algorithm for extracting salient edges. The method can result in an edge-preserving smoothing, which is well consistent with the human vision system; that is, human's perception of local details in an image is significantly weaker than that of salient targets. For license plate images, license characters are commonly designed to have high contrast, while the scene background mainly contains low contrast detail information. With the $L_{0}$-smoothing algorithm, the characters can be reserved, while the most low-contrast background would be filtered out. It has proved that such an operator is able to strengthen the license feature remarkably and suppress the background details significantly.

\subsection{Model}

Let $g$ be the source image and $f$ be target image in $\mathbb{R}^{N}$ space, respectively. The $L_{0}$-norm image smoothing can be formulated as the following minimization problem,

$$
\underset{f}{\arg \min } E(f)=\|f-g\|_{2}^{2}+\lambda\|\nabla f\|_{0},
$$

where $\nabla f$ is the gradient, $\|x\|_{2}$ is $L^{2}$-norm, and $\|x\|_{0}$ counts the number of non-zero elements of the vector $\mathrm{x}$, which results in a sparse constraint on gradient, ${ }^{1}$ and $\lambda$ is a weight controlling the significance of the two terms. For the 2-D discrete images, Eq. (1) can be rewritten as

$$
\underset{f}{\arg \min } E(f)=\sum_{i}\left(f_{i}-g_{i}\right)^{2}+\lambda C(f) .
$$

For the $i$-th pixel, the image gradient $\nabla f_{i}=\left(\partial_{x} f_{i}, \partial_{y} f_{i}\right)^{T}$ is calculated as the color difference between neighboring pixels along the $\mathrm{x}$ and $\mathrm{y}$ directions. Thus the term $C(f)$ can be expressed as

$$
C\left(f_{i}\right)=\left\{\begin{array}{l}
1,\left|\partial_{x} f_{i}\right|+\left|\partial_{y} f_{i}\right| \neq 0, \\
0,\left|\partial_{x} f_{i}\right|+\left|\partial_{y} f_{i}\right|=0 .
\end{array}\right.
$$

For color images, the gradient magnitude is defined as the sum of gradient magnitudes in RGB color space.

\subsection{Solver}

Notice that the $L_{0}$-norm minimization is difficult to solve because the pixel-wise difference in the model results in a nonsmooth convex optimization problem. Traditional gradient descent or other discrete optimization methods are not suitable for this case. Wang [20] presented a novel efficient alternating optimization strategy to solve a $L^{p}(p \geq$ 1)-norm convex problem. Xu [19] also adopted the similar half-quadratic splitting scheme, which decomposes the original problem into two convex sub-problems by introducing new auxiliary variables and then solves the original problem by solving the two subproblems iteratively. As shown in [19], the mode in Eq. (1) can achieve an amazing result by preserving the salient edge or structures and removing the tiny textures. We provide a brief introduction to the solution. The reader is referred for more details, to papers [19, 20].

Traditionally, we introduce two auxiliary variables $h_{i}$ and $v_{i}$, corresponding to $\partial_{x} f_{i}$ and $\partial_{y} f_{i}$, respectively, so that the objective function of Eq. (2) can be rewritten as

$$
\begin{aligned}
\min _{f, h, v} E(f) & =\sum_{i}\left(f_{i}-g_{i}\right)^{2}+\lambda C(h, v) \\
& +\beta\left(\left(\partial_{x} f_{i}-h_{i}\right)^{2}+\left(\partial_{y} f_{i}-v_{i}\right)^{2}\right),
\end{aligned}
$$

where $C(h, v)=1$ if $\left|h_{i}\right|+\left|v_{i}\right| \neq 0$, else 0 ; and parameter $\beta$ automatically controls the similarity between variables $(h, v)$ and their corresponding gradients. It can be seen

1 Note that this $L_{0}$-norm sparsity measure is not a true norm. 


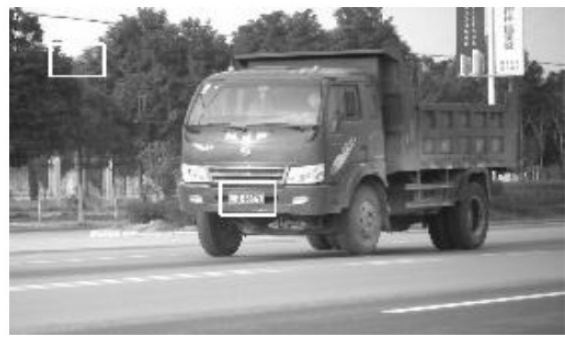

(a) Input image

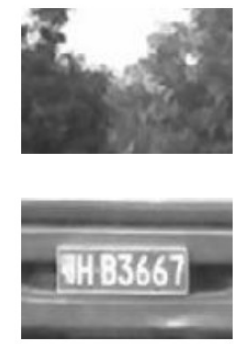

Figure 1: Image smooth using LOSmoothing algorithm.

that when $\beta \rightarrow+\infty$, the solution of Eq. (4) approximates to that of Eq. (2). Notice that Eq. (4) can be solved through alternatively minimizing $(h, v)$ and $f$. In each pass, one set of the variables is fixed with values obtained from the previous iteration.

Subproblem 1 computing $f$ : The subproblem of estimating $f$ corresponds to fixing $h_{i}$ and $v_{i}$ and minimizing the function,

$$
\min _{f} E(f)=\sum_{i}\left(f_{i}-g_{i}\right)^{2}+\beta\left(\left(\partial_{x} f_{i}-h_{i}\right)^{2}+\left(\partial_{y} f_{i}-v_{i}\right)^{2}\right) .
$$

The above function is quadratic and thus has a global minimum with the gradient descent method, by omitting the terms not involving $f$ in Eq. (4). In order to speed up the solution, $\mathrm{Xu}$ [19] introduced the fast Fourier transform (FFT), which transforms image gradient operator into the frequency domain and yields,

$$
f=\mathscr{F}^{-1}\left\{\frac{\mathscr{F}(g)+\beta\left(\mathscr{F}\left(\partial_{x}\right) \star \mathscr{F}(h)+\mathscr{F}\left(\partial_{y}\right)^{\star} \mathscr{F}(v)\right)}{\mathscr{F}(1)+\beta\left(\mathscr{F}\left(\partial_{x}\right)^{\star} \mathscr{F}\left(\partial_{x}\right)+\mathscr{F}\left(\partial_{y}\right)^{\star} \mathscr{F}\left(\partial_{y}\right)\right)}\right\},
$$

where $\mathscr{F}$ is the FFT operator, $\mathscr{F}^{-1}$ denotes its unitary adjoint, and $\mathscr{F}(1)$ is the Fourier Transform of the delta function. Notice that * is a component-wise multiplication operator. Compared to minimizing Eq. (5) directly in the image space, which involves a large-scale matrix inversion, computation in the Fourier domain is much faster due to the simple component-wise division.

Subproblem 2 computing $(h, v)$ : The objective function for $(h, v)$ is

$$
\min _{h, v} E(f)=\lambda C(h, v)+\beta\left(\left(\partial_{x} f_{i}-h_{i}\right)^{2}+\left(\partial_{y} f_{i}-v_{i}\right)^{2}\right),
$$

where $C(h, v)$ returns the number of non-zero elements in $\left|\partial_{x} f_{i}\right|+\left|\partial_{y} f_{i}\right|$. This apparently sophisticated sub-problem can actually be solved quickly, because the energy function of Eq. (7) can be spatially decomposed where each el-
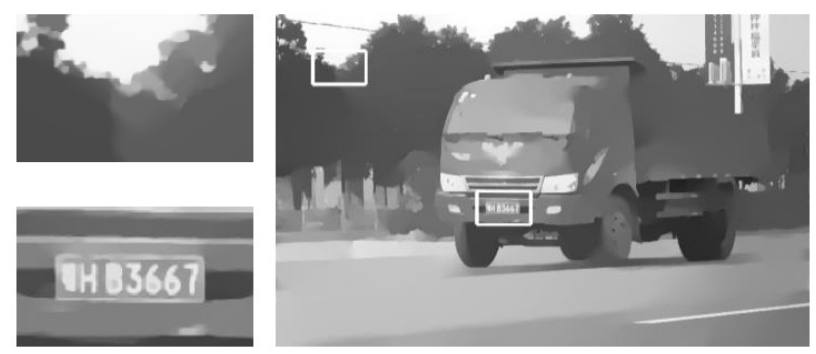

(b) $L_{0}$-norm smoothing result

ement $h_{p}$ and $v_{p}$ is estimated individually. It reaches its minimum $E^{\star}$ under the condition,

$$
h_{i}, v_{i}=\left\{\begin{array}{l}
(0,0),\left(\partial_{y} f_{i}\right)^{2}+\left(\partial_{y} f_{i}\right)^{2} \leq \frac{\lambda}{\beta}, \\
0, \text { otherwise. }
\end{array}\right.
$$

With the above analyses, the global optimum $E^{\star}$ of Eq. (2) can be obtained approximately by solving the two minimization problem alternatingly. The parameter $\beta$ is automatically adjusted in iterations, it starts from a small value $\beta_{0}$, multiplying by $\kappa$ in each iteration. Such a scheme is effective to speed up convergence. It meets the actual requirements well by setting $\beta_{0}, \beta_{\max }$ and $\kappa$ to $2 \lambda, 1 e 5$ and 2 in practical applications. As shown in Figure 1, where (a) is an image with trees and billboard and (b) is the smoothing result. It is clear that the multiple textures in the background are removed and the salient structures including the license plate are reserved by using the $L_{0}$-norm image smoothing algorithm.

\section{License location}

License plate location is designed to determine the location and size of licenses and extract them from images. By utilizing the $L_{0}$-norm image smoothing, the salient edges are reserved and the background textures can be remarkably removed. As shown in Figure 1, the long lines with low textural information can be filtered out easily. In the following sections, we present a simple but robust filtering scheme to select out the license plate. As it is difficult to determine the size of the license under changing circumstances, we thus employ a simple multi-scale analysis tool, binary image to solve the scale problem. Figure 2 shows the whole flow chart of our license location. 


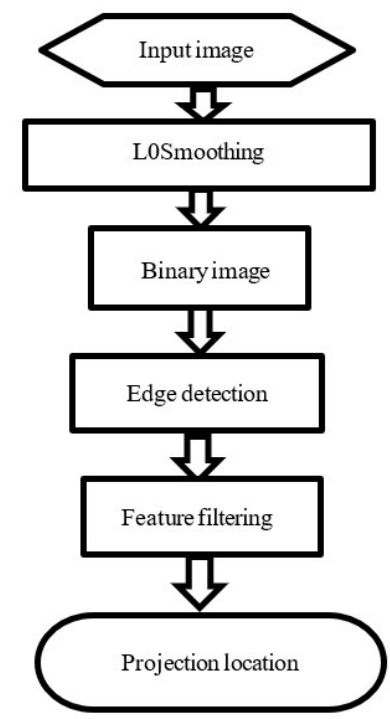

Figure 2: The flow chart of license plate location.

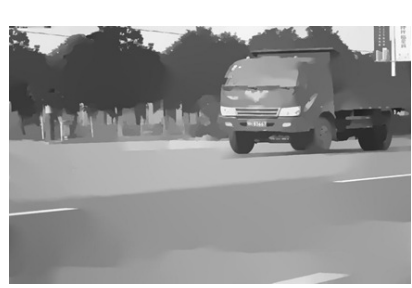

(a) $L_{0}$-norm smoothing result

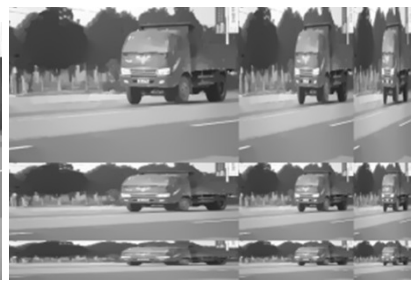

(b) Binarized image
Figure 3: An example of binarized image with 3 scales.

For license plates, the variation of the scale (size) is a key factor to be considered in the location algorithm. These variations can result from different aspects, for example, the angle of photographing, the distance between targets and camera, the movement of the target and so on. In such case, multi-scale analysis methods always give an effective way for image processing. Prevailing multi-scale analysis theory includes the pyramidbased [21] and wavelet-based [22] algorithms. Nevertheless, most of them are computational complexity or suffer from complex parameter-settings. To reduce the impact of scale variation, Cheng [23] introduced a simple binarized image. We take the sample configuration in succession of the $L_{0}$-norm image smoothing. The more details are discussed below.

\subsection{Binarized image}

Definition 1: Let $I$ be an image, and $I_{S}$ is resized from $I$ with a scale $s$, where $s \in S=\left\{s_{m, n}\right\}=\left\{2^{-m}, 2^{-n}\right\}_{m, n \in Z}$ and indexes $m$ and $n$ represent the row and column scales, respectively. If rearrange the new images $\left\{I_{s}\right\}_{s \in S}$ on a 2-D plane in the descending order of the scales $\left\{s_{m, n}\right\}$; then one can obtain a new image $B=\left\{I_{s}\right\}_{s \in S}$, called binarized image. In the context of the binarized image, a license plate with any scale can be resized to a pre-defined optimal scale $s_{0}$ by using this binarized-scaling, if there is discrepancy between the input and optimal licenses. The binarized image $B$ provides a multiscale analysis of the image $I$.

As shown in Figure 3, a multi-scale analysis based on the binarized-scaling is deduced, where (a) is the smoothed image and (b) is the binarized image. The row and column scales of the corresponding sub-diagram in (b) are 1/2,1/4 and 1/8 of the source size, respectively. Given that the license size, for example, in optimal scale $s_{0}$ with $180 \times 28$ pixels, then an image with a $300 \times 50$ pixels license can be found in the binarized image with a matching scale $s_{m, n}=\{0.5,0.5\}$, in which the license size is most close to the optimal size.

Moreover, the binarized scaling in the horizontal and vertical directions are independent. By combining different scales, the non-uniform scaling of images can be achieved, which is adapted to the different variations of the licenses, including tilt and deformation. Theoretically, no matter what size the license is, there is a resized subimage in the (b) that corresponds to the optimal scale.

As a multiscale analysis, the binarized image is simple and efficient, which also constitutes one of the cores of our whole scheme. In a practical application, a 2 to 4 scaledecomposition of the binarized image can be satisfied the requirements of a practical license plate location.

\subsection{Feature filtering}

As a salient target in images, a license plate can be quickly recognized by the human visual system, discriminating it from other targets in terms of the important features such as size, color, and textures. With the above configurations, a mass of local textural information in the background has been suppressed and the smoothed image has also been resized with an optimal scale by using the binarized image. In view of this, we further boost license feature with several rules as follows: 
(1) Geometrical structures: the license, characters and character strokes have a certain length and vary in a limited range in the optimal scale.

(2) Salient edges: a license plate consists of characters, letters and digits, and they have a high contrast compared with the background. In other words, the license contains abundant edges that can be reserved under the $L_{0}$-norm smoothing, in contrast to the multiple pseudo targets in the complex background.

(3) Textures: the abundant edges in a license formulate special textural information and the high density of edge points which can be used for location.

The three terms enable us to handle the problem in a rather flexible way. The term (1) interprets that the size of objects changes in a certain range, which motivates us to introduce "binarized image" to simplify the problem. (2) implies that the $L_{0}$-norm smoothing can preserve the abundant edge information in license region and remove the tiny edges in the background. The term (3) indicates that a license plate has rather high density of edge points, and hence can be used as a criterion for location. With the three priors, we first take $L_{0}$-norm smoothing and then give the binarized images. We, for example, assume the optimal size of characters (or letters and digits) in a license plate is $s_{0}=32 \times 16$ pixels and the scale varies in the range $\left(\frac{2}{3} s_{0}, \frac{4}{3} s_{0}\right)$. The feature filtering process can be concluded as follows:

Step 1: Edge extraction. Different algorithms can be used to extract the edge information of the binarized image, the "Sobel" edge extraction operator is a suitable one in terms of the effectiveness and efficiency.

Step 2: Removing long line. The contour $\mathrm{C}$ of the characters does not exceed 128 pixels under the optimal scale $s_{0}$, we set a threshold of $T=250$ pixels as the longest line of characters in consideration of the scale variation: $\left(\frac{2}{3} s_{0}, \frac{4}{3} s_{0}\right)$. We directly remove the long lines whose length is large than this threshold.

Step 3: Structure analysis. The length of strokes of the characters is limited in a certain range along the horizontal and vertical directions and does not exceed a certain value. In the optimal scale $s_{0}$, we set the horizontal threshold $T_{h}=24$ pixels and vertical threshold $T_{v}=48$ pixels, respectively.

Step 4: Texture density analysis. The abundant edges in a license plate give a high density of edge points, which stands out clearly in succession of step $1 \sim$ step 3 . Taking a window $\omega$, for example $48 \times 240$ pixels, into account, we choose the central point as a candidate license point with a threshold $T_{d}=0.5^{\star} \omega$ in the window, otherwise, this point belongs to non-license plate.

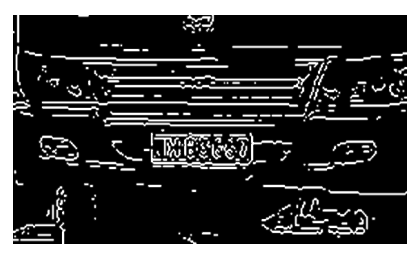

(a) Edge extraction

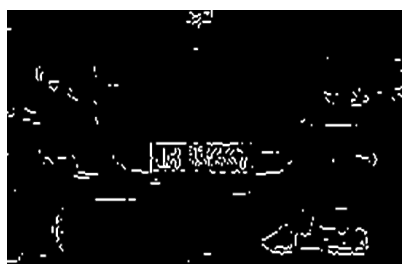

(c) Structure analysis

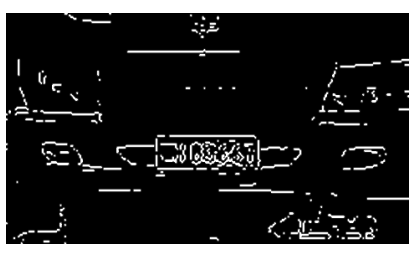

(b) Removing long lines

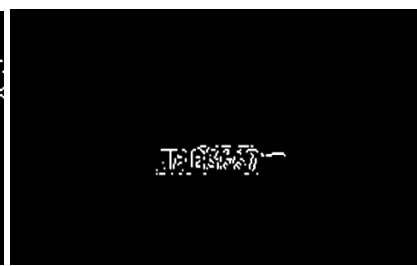

(d) Texture density analysis
Figure 4: The results of feature filtering.

As shown in Figure 4, where (a) is the edge map using the "Sobel" edge extraction operator, and there are amount of edge points in grids, car lights, and outlines of the vehicle; (b) is the result of removing long line, the most outline edges of the car body are filtered out; (c) and (d) are the results using structural and textural analysis, respectively, where the interference of edge points in background is further weakened and the license object is highlighted eventually. With the above steps, a large number of pseudo-targets of can be filtered out, while the license plates are more discriminate in one by one step.

Experimental results illustrate that the interference of the complex background can be significantly weakened by combining the "edge-aware smoothing", binarized image and feature filtering, which reduces the complexity of the license location algorithm, especially in the complex circumstance.

\subsection{Projection}

In general, with the above steps, the license plates, as shown in Figure 4 (d), are stand out in an image despite some few edge points around the targets. In this case, a simple projection-based method is introduced to locate the license plates. Taking both horizontal and vertical directions into account, the distributions of the projection histograms are summarized as follows:

(1) The license plate commonly has 7 characters, in consideration of the influence of the license outlines, the variation of circumstance illumination and other factors, the number of edge points in each horizontal line is from 


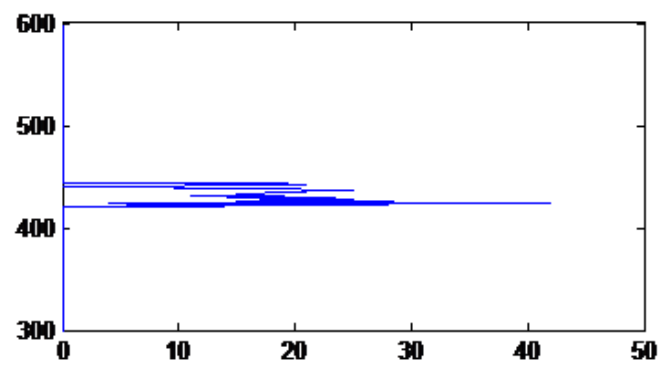

(a) Horizontal projection

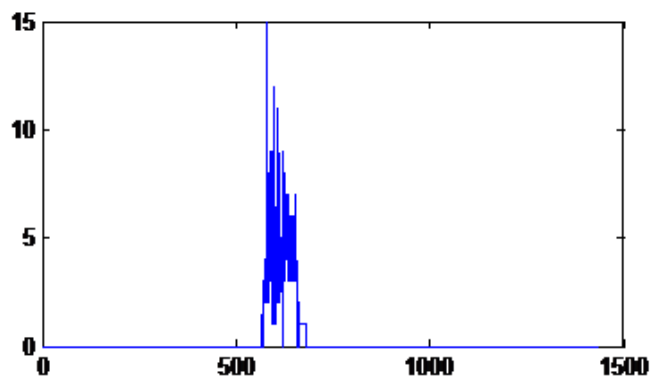

(b) Vertical projection

Figure 5: The horizontal and vertical projection histograms.
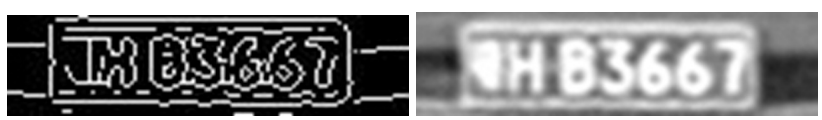

Figure 6: The extracted license plate object.

15 to 60 pixels in most cases. The one in the vertical direction is generally between 10 and 30 pixels.

(2) In the vertical projection, the width between two peaks generating by the neighbor characters is generally between 10 and 20 pixels.

The horizontal and vertical projection histograms, as shown in Figure 5, produce clear peaks. In practice, we use a weighted sum of the projection histogram to improve the continuity and steepness of the peaks in the histograms. Given the source histogram $H$, a simple weight kernel $w=$ $[0.1,0.2,0.4,0.2,0.1]$ is used to modify the histogram, which gives a new histogram $H_{1}$, satisfying,

$$
H_{1}=w^{\star} H \text {. }
$$

In addition, the number of the jumped edge of the license can be used to delete the false license plate targets. Figure 6 shows the extracted license plate, where (a) is the edge image and (b) is the corresponding grayscale license plate image.

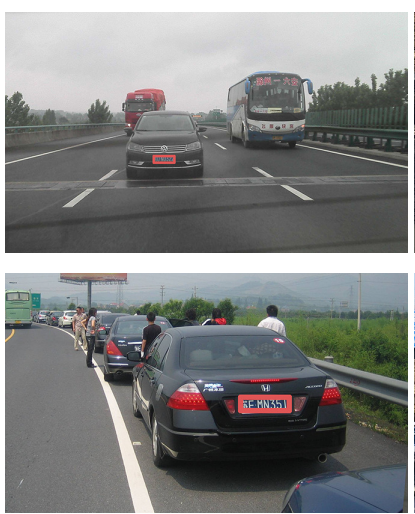

(a) Simple mode
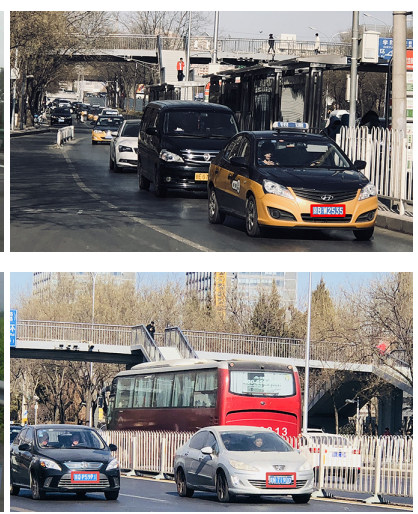

(b) Complex mode
Figure 7: Our license plate location results.

\section{Experimental results}

We will evaluate the performance of our algorithm in this section. In order to verify the accuracy of the algorithm, a dataset with 1000 license plate images is collected, which consists of 700 images with simple background (highway) and 300 images captured in cites with complex backgrounds including buildings, people and landscapes. Our basic algorithm is implemented in MATLAB 2015b without special optimization. It takes about $2 \sim 3$ seconds, spending most computation time in $L_{0}$-norm smoothing, to deal with a $768 \times 396$ resolution image on a desktop PC with Intel i7 $2.80 \mathrm{GHz}, 16 \mathrm{~GB}$ RAM, and Win 10 64bit OS.

In all the experiments, a three-levels' binarized image analysis is adopted and we assume the optimal license size in scale $s_{0}$ is $180 \times 28$ pixels. Theoretically, a license size with a scale between $s_{0}$ and $4^{\star} s_{0}$ can be located by the proposed method. As shown in Figure 7, two representative cases are processed, where (a) is a simple mode with highway background, and (b) has complex background, including trees, buildings and people. In the total of 1000 test images, 29 images are failed to detect out the license target, 4 of which are the simple mode (highway) and the other images are sampled from the difficult mode (city). Besides, 20 images are located incorrectly, most of which are captured with city background.

We quantitatively evaluate our method with method with existing ones, including the Top-hat method [25], a fusion method based on the Maximally Stable Extremal Regions (MSER) and SIFT [11], Wavelet-based method [26] and Convolutional Neural Network (CNN)based method [15]. The detector is evaluated in terms of three criterions: precision, recall and $F_{1}$-score rate. The 
Table 1: Quantitative evaluation of our algorithm.

\begin{tabular}{c||c|c|c}
\hline Method & Precision(\%) & Recall (\%) & $F_{1}$-score(\%) \\
\hline \hline Top-hat [25] & 77.50 & 84.50 & 88.61 \\
MSER \& SIFT [11] & 83.73 & 90.47 & 86.97 \\
Wavelet [26] & 90.30 & 94.03 & 95.00 \\
CNN-based [15] & 97.80 & 95.30 & 96.01 \\
Ours & 96.10 & 95.40 & 95.30 \\
\hline
\end{tabular}

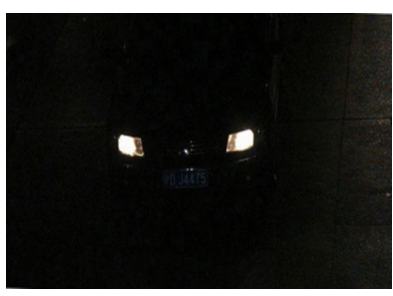

(a) Low-lighting image

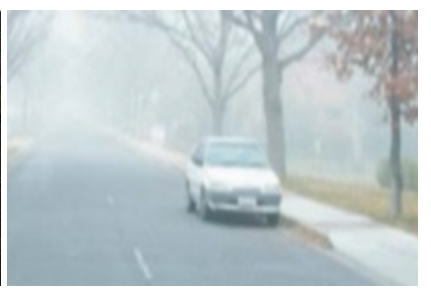

(b) Haze image
Figure 8: The degraded low-lighting and haze images.

precision is defined as the ratio of the number of license plates that are correctly divided to the total number of license plates. The recall represents the fraction of the true objects that were found. The $F_{1}$-score is given by Eq. (10). The statistical results are shown in Table 1. As we can see, our algorithm produces competitive or superior results compared to the other methods despite the CNNbased method [15].

$$
F_{1} \text {-score }=2 \times \frac{\text { precesion } \times \text { recall }}{\text { precesion }+ \text { recall }} .
$$

For the failed cases, the main reason is that the contrast between the license plate and background is too low, and the local structures of the characters are removed by the $L_{0}$-norm smoothing, resulting in poor edges. Two typical cases are shown in Figure 8, where (a) is captured in the low-lighting environment, and the license plate is extremely and almost unidentifiable by the naked eye; and (b) is an image heavy with fog and the license plate is significantly blurred. In fact, for the case (a), a contrast enhancement algorithm can be adopted before location; for the case (b), a dehazing method [24], can be used as a preprocessing method in practice.

\section{Conclusion}

In this paper, we propose a simple license plate location method, which is based on the L0-norm image smoothing and feature filtering. The method reduces the occurrence of pseudo-licenses as it can suppress the complex background textures significantly. By using a series of feature filtering operators, the proposed method highlights the license plate target and weakens the interference factors in the complex background continuously, which reduces the complexity of the license plate location algorithm. In practice, the proposed method can achieve a high accuracy result, which gives a basis for the subsequent characters segmentation and recognition of the license plate recognition system. For low contrast situations such as low-lighting and fog cases, the location algorithm has a high failure rate. In future work, we attempt to improve the accuracy of the license plate location in such cases.

\section{References}

[1] Du S., Ibrahim M., Shehata M. S., Badawy W. M., Automatic License Plate Recognition (ALPR): A state-of-the-art review, IEEE Transactions on Circuits and Systems for Video Technology, 2013, 23(2), 311-25

[2] Wang T. H., Ni F. C., Li K. T., Chen Y. P., Robust license plate recognition based on dynamic projection warping, IEEE International Conference on Networking, Sensing and Control, 2004, 2, 784-788

[3] Lee H. J., Chen S. Y., Wang S. Z., Extraction and recognition of license plates of motorcycles and vehicles on highways, In: Proceedings of the 17th International Conference on Pattern Recognition, 2004, ICPR 2004, 4, 356-359

[4] Zheng D., Zhao Y., Wang J., An efficient method of license plate location, Pattern Recognition Letters, 2005, 26(15), 2431-2438

[5] Wu Y., Liu S., Wang X., License plate location method based on texture and color, In: 2013 IEEE 4th International Conference on Software Engineering and Service Science, 2013, 361-364

[6] Lee E. R., Kim P. K., Kim H. J., Automatic recognition of a car license plate using color image processing, In: Proceedings of 1st International Conference on Image Processing, 1994, 2, 301-305

[7] Shi X., Zhao W., Shen Y., Automatic license plate recognition system based on color image processing, International Conference on Computational Science and Its Applications, 2015, 1159-1168

[8] Abolghasemi V., Ahmadyfard A., An edge-based color-aided method for license plate detection, Image and Vision Computing, 2009, 27(8), 1134-1142

[9] Huang S., The research and achievement of vehicle plate recognition, Phd thesis, Si Chuan University, Si Chuan, China, 2005

[10] Chen Z. X., Liu C. Y., Chang F. L., Wang G. Y., Automatic licenseplate location and recognition based on feature salience, IEEE Transactions on Vehicular Technology, 2009, 58(7), 3781-3785 
[11] Lim H. W., Tay Y. H., Detection of license plate characters in natural scene with MSER and SIFT unigram classifier, In: 2010 IEEE Conference on Sustainable Utilization and Development in Engineering and Technology, 2010, 95-98

[12] Kim K. K., Kim K. I., Kim J. B., Kim H. J., Learning-based approach for license plate recognition, In: Neural Networks for Signal Processing X, Proceedings of the 2000 IEEE Signal Processing Society Workshop, 2000, 2, 614-623

[13] Zhang H., Jia W., He X., Wu Q., Learning-based license plate detection using global and local features, In: 18th International Conference on Pattern Recognition, 2006, 2, 1102-1105

[14] Hu C., Bai X., Qi L., Chen P., Xue G., Mei L., Vehicle color recognition with spatial pyramid deep learning, IEEE Transactions on Intelligent Transportation Systems, 2015, 16(5), 2925-2934

[15] Selmi Z., Halima M. B., Alimi A. M., Deep learning system for automatic license plate detection and recognition, In: 2017 14th IAPR International Conference on Document Analysis and Recognition (ICDAR), 2017, 1132-1138

[16] Polishetty R., Roopaei M., Rad P., A next-generation secure cloud-based deep learning license plate recognition for smart cities, In: 2016 15th IEEE International Conference on Machine Learning and Applications (ICMLA), 2016, 286-293

[17] Krizhevsky A., Sutskever I., Hinton G. E., ImageNet classification with deep convolutional neural networks, In: Advances in Neural Information Processing Systems, 2012, 25, 1097-1105
[18] LeCun Y., Yoshua B., Geoffrey H., Deep Learning, 2016

[19] Xu L., Lu C., Xu Y., Jia J., Image smoothing via L0 gradient minimization, ACM Transactions on Graphics, 2011, 30(6), Article 174

[20] Wang Y., Yang J., Yin W., Zhang Y., A new alternating minimization algorithm for total variation image reconstruction, Siam Journal on Imaging Sciences, 2008, 1(3), 248-272

[21] Paris S., Hasinoff S. W., Kautz J., Local Laplacian filters: Edgeaware image processing with a Laplacian pyramid, ACM Transactions on Graphics, 2011, 30(4), 68

[22] Daubechies I., The wavelet transform, time-frequency localization and signal analysis, IEEE Transactions on Information Theory, 1990, 36(5), 961-1005

[23] Cheng M. M., Zhang Z. M., Lin W. Y., BING: Binarized Normed Gradients for Objectness Estimation at $300 \mathrm{fps}$, In: Proceedings of the 2014 IEEE Conference on Computer Vision and Pattern Recognition, 2014, 3286-3293

[24] He K., Sun J., Tang X., Single image haze removal using dark channel prior, IEEE Transactions on Pattern Analysis and Machine Intelligence, 2011, 33(12), 2341-2353

[25] Martin F., Garcia M., Alba J. L., New methods for automatic reading of VLP's (Vehicle License Plates), In: Proceedings of IASTED International Conference SPPRA, 2002, 26(6), 257-271

[26] Yu S., Li B., Zhang Q., Liu C., Meng M. Q. H., A novel license plate location method based on wavelet transform and EMD analysis, Pattern Recognition, 2015, 48(1), 114-125 